\title{
EL CONCEPTO DE COMPETENCIAS Y SU ADOPCIÓN EN EL CONTEXTO UNIVERSITARIO
}

\author{
MARÍA JOSÉ GARCÍA-SAN PEDRO ${ }^{1}$
}

Departamento de Pedagogía Aplicada. Facultad de Ciencias de la Educación. Universidad Autónoma de Barcelona.

\section{RESUMEN}

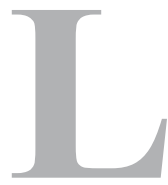

a formación por competencias se ha transformado, en términos de diseño y desarrollo curricular, en el eje del cambio educativo. Este cambio, en el enfoque curricular, reorienta las decisiones didácticas en relación con la enseñanza, aprendizaje y evaluación, centrándolo en el estudiante, sus procesos y resultados de aprendizaje.

Esta aportación considera aspectos conceptuales en relación con la definición de competencias, sus consecuencias, fundamentos y concreción en la Educación Superior. Las reflexiones que se presentan remiten a los fundamentos ontológicos y epistemológicos de la temática y se proyectan sobre la dimensión organizativa de las titulaciones a través de un estudio de casos múltiple. La metodología cualitativa y el enfoque hermenéutico posibilitan acceder a la concepción de competencias y a la percepción del cambio de paradigma en el profesorado implicado en la transformación del modelo universitario español.

Palabras clave: competencias, Educación Superior, trazabilidad, cambio, innovación; enseñanza-aprendizaje, epistemología, metodología cualitativa, estudio de casos.

\section{ABSTRACT}

In terms of design and curriculum development, competences-based training is at the heart of how education is changing in Spain in terms of teaching, learning and assessment, with the focus now on students and their learning processes and results.

This paper considers conceptual aspects relating to how competences are defined, and what the consequences and practical consequences are in Higher Education. This paper uses a multiple case study to make a series of ontological and epistemological reflections with particular regard to qualifications. The qualitative approach and interpretative method

\section{CORRESPONDENCIA:}

María José García-San Pedro

Departament de Pedagogia Aplicada. Facultat de Ciències de l'Educació

Universitat Autònoma de Barcelona

Edifici G-6. Campus de Bellaterra · 08193 CERDANYOLA DEL VALLÈS (Barcelona) España

Tel: +34 $935814836 \cdot$ Móvil: $696126085 \cdot$ mariajose.garcia.san.pedro@uab.cat

1 Con el apoyo de la Generalitat de Cataluña y el Fondo Social Europeo. 
allow for an understanding of competences and the perception of the paradigm shift in the teaching staff involved in transforming the Spanish university model.

Key words: competences, learning outcomes, Higher Education, change, mapping, curricular innovations, teaching and learning, epistemology, qualitative research, case study.

\section{CONTEXTUALIZACIÓN}

El Espacio Europeo de Educación Superior (EEES), a partir de su inicio oficial en 1999 con la "Declaración de Bolonia", ha dado lugar a un proceso de cambio en algunas universidades europeas. Este proceso ha tenido dos objetivos principales, por un lado, alimentar una economía basada en el conocimiento y, por el otro, consolidarse como una opción atractiva para la comunidad científica internacional. En este contexto, ya no se habla de la necesidad de innovación, sino de cuáles son sus estrategias y componentes.

En el contexto español se han llevado a cabo diversas iniciativas, lideradas por organismos estatales, autonómicos, agencias de calidad y las mismas universidades, con la finalidad de incorporar los desafíos y los objetivos del Proceso de Bolonia. Ejemplo de estas iniciativas son, entre otras, los Libros Blancos de la ANECA y los Planes Piloto de Adaptación que han puesto en marcha algunas comunidades autónomas -como Cataluñay las "Redes de Investigación Docente" y "Redes de Estudio de la Docencia de Calidad en Trabajo Social" que ha puesto en marcha la Universidad de Alicante. Estas iniciativas han favorecido, fundamentalmente, el intercambio de experiencias y la comunicación entre la universidad, sus graduados y el mundo laboral.

En este escenario, surge el discurso de las competencias como el lenguaje capaz de responder a la movilidad, la transferencia, el reconocimiento de los aprendizajes y la internacionalización de la Educación Superior. Por otra parte, debido a sus orígenes en el mundo laboral y, en tanto sistema de reconocimiento de cualificaciones, este modelo no es nuevo ni innovador, incluso tiene acreditados referentes en la esfera de la formación profesional. Sin embargo, esta trayectoria temporal no siempre se traduce en clarificación conceptual.

Esta aportación se propone profundizar en los antecedentes conceptuales de las competencias en el ámbito de la Educación Superior, delimitar aquellos rasgos que deberían caracterizar específicamente a las competencias universitarias y conocer la concepción de competencias y la visión del cambio en el modelo formativo que tiene el profesorado implicado en la transformación del modelo universitario español.

\section{EVOLUCIÓN CONCEPTUAL EN EL ÁMBITO ACADÉMICO}

El movimiento de las competencias ha asumido diferentes nombres en el contexto internacional de los últimos veinte años. Puede decirse que esta evolución es su respuesta a las crecientes necesidades y demandas de los procesos de evaluación de calidad. Entre las denominaciones halladas se pueden citar: "Graduate attributes Movement" (Chanock, 2003), "Generic Graduate Attributes" (Barrie, 2005, 2006), "Graduate Skills" (Chanock, 2004), "Generic Capabilities" (Bowden et al. 2002), "personal transferable skills" (Drummond et al. 1998), "Graduate capability development" (Kift, 2002). Si bien con distintos matices, todas las corrientes generadas coinciden en vincular la enseñanza universitaria con el mundo laboral y en ofrecer una dimensión más transparente de la evaluación vinculada al reconocimiento de cualificaciones y a la movilidad. 


\subsection{Conceptualizaciones previas}

Existen conceptos muy cercanos al término competencia que no deben prestarse a confusión. El reconocimiento de estas diferencias puede realizarse a través de autores como Tobón (2004) y Fernández-Salinero Miguel (2006):

- Cualificaciones profesionales: término que se ha empleado para referirse a la capacidad general de desempeñar todo un conjunto de tareas y actividades relacionadas con un oficio y apoyadas en una certificación acreditativa. Las competencias, a diferencia de las cualificaciones, enfatizan su desempeño en procesos personales y profesionales específicos, donde lo central es la adaptación al cambio y la flexibilidad en el desempeño de actividades. Las cualificaciones profesionales serían, por tanto, un elemento previo para la definición de las competencias.

- Capacidades: condiciones cognitivas, afectivas y psicomotrices fundamentales para aprender y que denotan la dedicación a una tarea. Son el desarrollo de las aptitudes. Las competencias, en este sentido, tienen como uno de sus componentes las capacidades con el fin de llevar a cabo una actividad.

- Destrezas: mediadoras entre las capacidades y las habilidades y cuya adquisición supone el dominio tanto de la percepción frente a los estímulos como de la reacción eficaz para ejecutar la tarea. La eficacia y la flexibilidad son dos cualidades que las definen. Las competencias tienen las destrezas como base para la actuación, pero difieren de éstas en que integran el conocimiento, los procedimientos y las actitudes en la búsqueda de objetivos a corto y largo plazo.

- Habilidades: consisten en cualidades que permiten realizar tareas y actividades con eficacia y eficiencia. Las competencias buscan también la eficiencia y la eficacia, pero además integran la comprensión de la situación, conciencia crítica, espíritu de reto, responsabilidad por las acciones y desempeño basado en indicadores de calidad.

- Actitudes: disposiciones afectivas para la acción que constituyen el motor que impulsa al comportamiento en los seres humanos. Inducen igualmente a la toma de decisiones y a desplegar un determinado tipo de comportamiento acorde con las circunstancias del momento. A este respecto, las competencias se componen de cuatro saberes: saber conocer, saber hacer, saber estar y saber ser. El saber ser, a la vez, está integrado por valores, estrategias psico-afectivas y actitudes. Por lo tanto, las competencias son un proceso de actuación amplio y las actitudes uno de sus componentes.

Otros conceptos que pueden guardar relación con la competencia, pero que no son sinónimos de ella, son los rasgos de personalidad y las aptitudes. Las aptitudes son las capacidades y disposiciones para el buen desempeño, pero no necesariamente remiten al logro o realización efectiva en una situación determinada, es decir, tienen una dimensión potencial. Los rasgos de personalidad permiten caracterizar a los individuos y explicar la variación de sus comportamientos en la ejecución de tareas específicas, mientras que las competencias afectan a la puesta en práctica integrada de aptitudes, rasgos de personalidad y también conocimientos adquiridos para cumplir bien una tarea compleja en un contexto determinado y dentro del espíritu de una estrategia y cultura identificadas (Levy-Leboyer, 1997).

Una diferenciación más que tiene que ver con la importancia que adquiere en el concepto de competencia la "experiencia personal". Ésta enriquece la actualización, refuerza la dimensión de expresión artística y de creación personal. Ocurre que, a diferencia de los saberes conceptuales y procedimentales, el saber experiencial es privado y pertenece al sujeto en el encuentro con la práctica misma. Este rasgo, para el ámbito educativo, es crucial, pues permite contemplar la vertiente individual del educando. 


\subsection{Hacia una definición operativa de las competencias}

En el camino hacia una definición operativa de las competencias, se hace necesario diferenciar algunos conceptos más (Kennedy, Hyland \& Ryan, 2006):

- Objetivos generales (intencionalidades): es una generalización amplia de una intención educativa. Indica lo que el profesor pretende cubrir en un conjunto de aprendizaje. Los objetivos están escritos generalmente desde el punto de vista del profesor para indicar el contenido general o la dirección del módulo o la unidad. Por ejemplo, introducir a los estudiantes en los principios básicos de la estructura atómica o proveer una idea general de la historia irlandesa del siglo XX.

- Objetivos: correspondientes a un módulo o programa, son afirmaciones específicas de las intenciones de la enseñanza. Por ejemplo, pretende cubrir un área específica de un bloque de aprendizaje: que los estudiantes entiendan el impacto y los efectos de los comportamientos y estilos de vida tanto en el medio ambiente local como global.

- Competencia: "Una combinación dinámica de atributos, habilidades y actitudes" ECTS User's Guide (2005). Agrega que las competencias se forman de diversas maneras y se evalúan en escala diferente. Pueden dividirse en específicas de un área o campo de estudio y en genéricas, es decir, comunes a cualquier curso de titulación.

- Resultado o logro de aprendizaje: ha resultado ser más útil y frecuente, dada la falta de acuerdos conceptuales con respecto al término "competencia". Describe, en términos cuantitativos o cualitativos, lo que se espera que el estudiante sepa, comprenda o sea capaz de demostrar al finalizar un módulo o un programa. Cada resultado es unívoco, debe describir un sólo logro, ser claro y fácilmente comprensible por toda la facultad. Debe ser redactado desde el punto de vista del estudiante.

En ocasiones, una de las mayores dificultades para conceptualizar con precisión las competencias es la confusión entre los conceptos anteriormente señalados, puesto que en su formulación se entremezclan las aproximaciones centradas en la enseñanza -punto de vista del docente- y las centradas en los resultados de los aprendizajes.

Hager, Holland y Becket (2002) establecen una definición de competencias que le otorga un status propio, al diferenciarlas del conocimiento profesional específico de cada área o disciplina y de las habilidades técnicas propias de la formación superior.

"Es un término ampliamente usado para designar "un rango de cualidades (qualities) y capacidades (capacities) que son consideradas crecientemente como importantes para la educación superior." Incluye habilidades de pensamiento (razonamiento lógico y analítico, solución de problemas, curiosidad intelectual), habilidades de comunicación efectiva, trabajo en equipo y capacidades para identificar, acceder y gestionar el conocimiento y la información; atributos personales como la imaginación, la creatividad y el rigor intelectual; y valores como la ética práctica (deontología profesional), persistencia, tolerancia e integridad. Esta colección de cualidades y capacidades tan diversas se diferencia del conocimiento profesional específico y las habilidades técnicas tradicionalmente asociadas con la educación superior.” (Hager et al. 2002:3)

Puede observarse que en esta conceptualización entran en juego cualidades y capacidades genéricas pertenecientes a distintos ámbitos: cognitivo, personal, interpersonal, de gestión del conocimiento, ético y volitivo.

La variedad de matices conceptuales puede observarse al destacar la dimensión originaria, única, de la persona que logra una competencia "descifrando" las claves del contexto y "combinando" saberes a través de una síntesis personal:

“(La competencia es)... una actuación originaria de la persona que integra su ser y sus saberes en la capacidad de enfrentarse a contextos de incertidumbre resolviendo con éxito sus demandas. (García San Pedro, 2007, p. 78) 
Pero ¿de qué modo se da la competencia? ¿Cómo se relacionan sus componentes? Se entiende que la competencia como constructo con antecedentes complejos es "una manifestación transversal de los componentes actitudinal, técnico, procedimental y social", que si no confluyen estos cuatro componentes no podríamos estar hablando del logro de una competencia, sino de un componente particular. Es precisamente este rasgo el que demanda el cambio curricular. Ya no puede hablarse de un contenido adquirido o no, sino de una aplicación contextualizada, transferida a una situación de aprendizaje-evaluación concreta. Concretamente, no se trata de saber si un estudiante de Química es capaz de establecer los compuestos de determinada fórmula -pongamos, por ejemplo, de un perfume o esencia- sino de saber si resuelve el problema de por qué una esencia o perfume es más caro que otro, qué variables intervienen y de qué forma (en su composición, elaboración, producción y venta) y cuál sería aconsejable utilizar según el objetivo que se pretenda.

\subsection{Clasificación de las competencias según su alcance y/o nivel de abstracción}

Es frecuente encontrar en la literatura específica que la falta de acuerdo conceptual es un obstáculo para la generalización del empleo del término competencias. Como ejemplo es sabido que existen tantas clasificaciones como estudios del tema. En este caso, proponemos una clasificación según el alcance y/o nivel de abstracción que tenga la competencia. Esto permitirá, operativamente, delimitar el tipo de competencias que se abordarán.

En consecuencia, se habla de cuatro niveles de competencias:

- Competencias básicas o instrumentales. Son aquellas asociadas a conocimientos fundamentales -que, normalmente, se adquieren en la formación general, básica u obligatoria- enfocadas a la comprensión y resolución de los problemas cotidianos y que permiten, posteriormente, el ingreso al trabajo. Por ejemplo, la comunicación oral, la escrita, la lectura o el cálculo.

- Competencias genéricas, transversales, intermedias, generativas o generales. Se relacionan con capacidades, atributos, actuaciones y actitudes amplias, transversales a distintos ámbitos profesionales. Podemos citar la capacidad para trabajar en equipo, el saber planificar o la habilidad para negociar.

- Competencias especificas, técnicas o especializadas. Se relacionan con aspectos técnicos directamente vinculados con la ocupación y las competencias específicas de una determinada área de estudio, que no son tan fácilmente transferibles a otros contextos laborales o académicos. Entre ellas, podemos encontrar la operación de maquinaria especializada, la formulación de proyectos de infraestructura, la elaboración de mapas cartográficos o la interpretación de variables climáticas.

- Meta-competencias, meta-qualities o “meta-skills". Son competencias genéricas, de alto nivel, que trascienden a otras competencias y que parecen favorecerlas, mejorarlas o posibilitar la adquisición de otras. Generalmente, se basan en la introspección, la meta-cognición, la auto-evaluación, el análisis de problemas, la creatividad, y el auto-desarrollo.

Como vemos, el alcance o abstracción como criterio de clasificación permite determinar los ámbitos de incidencia de las competencias. De esta manera, por ejemplo, a nivel de una asignatura, confluirán distintas competencias, unas de ámbito genérico y otras específicas de la asignatura y de la titulación.

Una aclaración que se hace necesaria es la relación que existe entre competencias genéricas y meta-competencias. Como se ha hecho referencia anteriormente, las meta-competencias son un tipo -más abstracto- de competencias genéricas. Comparten con éstas su naturaleza transversal y su carácter genérico, pero la combinación -de componente fundamentalmente intelectual- necesaria para adquirirla o actualizarla requiere un nivel 
mayor de madurez intelectual, un ejercicio combinatorio de las destrezas intelectuales implicadas, alta intervención del yo y alta capacidad de descentración. Por ejemplo, mientras que una competencia genérica podría ser la planificación de un proceso $x$ o el diseño de un modelo y, una meta-competencia sería la capacidad para detectar las debilidades del modelo o del diseño realizado, los momentos críticos de un proceso o la previsión o anticipación de fallos en el sistema y el diseño de soluciones alternativas.

\section{LAS COMPETENCIAS EN EL ÁMBITO UNIVERSITARIO}

Bowden et al. (2002) señalan algunas diferencias o condiciones propias de este tipo de competencias en el ámbito universitario:

- Son acordadas por una "comunidad universitaria".

- Para ser aprendidas y evaluadas durante el tiempo en el que los estudiantes estén en la universidad.

- La selección va más allá del conocimiento disciplinar.

- Preparan a los graduados como "agentes del bien social en un futuro desconocido".

Estos cuatro referentes concretan las diferencias específicas que puede tener el tratamiento de las competencias en el ámbito universitario: deben partir del consenso y la participación de todos los miembros de la comunidad universitaria, por lo cual se vincula el mundo académico con el profesional, por la influencia de los participantes en la definición; deben "garantizarse" - tanto en su enseñanza como en su evaluación-durante el tiempo de permanencia como estudiantes; atraviesan el conocimiento disciplinar -por tanto, no responderían a una única disciplina-; y vinculan al futuro graduado al desempeño de su profesión y su rol como ciudadano a través del ejercicio de la dimensión deontológica de su profesión. Estas condiciones deben darse de tal modo que, en la definición de competencias que se formule en una titulación, se expliciten sus fundamentos. Estos fundamentos definen las concepciones en relación al ser humano, a los valores, al conocimiento y las formas de aprehenderlo.

Además de las implicaciones de los aspectos señalados por Bowden et al. (2002), agregamos algunas notas distintivas más:

- Una competencia universitaria ES...

- Un desempeño/actuación concreto y global en una determinada área del saber.

- Una respuesta o forma de ver o comprender el objeto de estudio de modo transversal, lo que posibilita dialogar con otras áreas o disciplinas y contemplarlas como posibilidad en la resolución del problema (transversalidad horizontal).

- A la vez, permite profundizar en el concepto (adquirir otros saberes propios del área de conocimiento), generando nuevos aprendizajes de mayor profundidad (por ejemplo, los adquiridos en especializaciones posteriores como el doctorado).

- Una competencia universitaria NO ES...

- Una adquisición de saberes, destrezas o conductas básicas, adquiribles en etapas previas de escolarización y necesarias para el desempeño ciudadano básico (lectura -leer un diario-, escritura -conocer el alfabeto-, cálculo -aplicar las operaciones matemáticas-).

- La habilidad profesional concreta e instrumental, adquirible con la práctica y descontextualizada -o independiente- de sus fundamentos.

- Una afirmación cerrada sobre lo que el estudiante será capaz de hacer a lo largo de su vida personal y profesional. 
- Un saber demostrable que no pueda medirse o captarse para su evaluación en el contexto curricular de la titulación, pues, entre otras razones, la formación universitaria requiere dar cuentas de los saberes adquiridos por sus estudiantes.

En consecuencia, cabe preguntar ¿qué clasificación operativa de competencias puede establecerse? Se entiende que, para el ámbito universitario, lo más frecuente es el empleo de los tres tipos de competencias: a) competencias nucleares, que corresponden a las que son propias de la universidad, otorgan la impronta y el sello de identidad institucional; b) competencias transversales, que son las que atraviesan a varias disciplinas $\mathrm{y}$, consecuentemente, deben desarrollarse a través del trabajo conjunto de ellas y c) competencias especificas, que son las propias del área, la titulación o la asignatura, otorgan lo propio y distintivo de la profesión (Gairín et al., 2009a:18).

Cabe destacar que, a través de la selección que realice la titulación, es decir, del "mapa de competencias" que quede configurado, es posible "leer" los fundamentos antropológicos, ontológicos y epistemológicos que acompañan la formación del graduado (García San Pedro, 2007). El mapa que quede definido puede constituirse en un indicador del cambio en la formación universitaria.

\subsection{Algunas condiciones para facilitar la adquisición de las competencias}

Es posible establecer algunas condiciones que facilitan la adquisición de las competencias a través de los módulos y áreas disciplinares:

- El conocimiento de lo que implica la competencia demandada (saber qué): el profesor debe tener claro qué saberes entran en juego y debe comunicarlo explícitamente al estudiante, para favorecer su participación, su implicación. El estudiante debe ser consciente de cuál es la demanda, qué saberes están implicados para actualizarlos y responder al contrato de aprendizaje que se establece.

- Conocimiento y dominio de las reglas y procedimientos implicados en la competencia demandada (saber cómo): las metodologías de enseñanza y evaluación deben estar en consonancia para favorecer los aprendizajes (alineamiento constructivo). La explicación permanente, por parte del profesor, de las destrezas que entran en juego en cada situación de aprendizaje facilita a los estudiantes que activen procedimientos metacognitivos, favoreciendo sus desempeños.

- Una actitud favorable por parte del estudiante para actualizar y transferir las competencias a otros contextos: se corresponde con los fundamentos del aprendizaje significativo.

- Una propuesta didáctica que promueva la ejercitación y transferencia de las competencias (escenario): es importante, en el conocimiento de las metodologías de enseñanza y evaluación, saber y seleccionar aquéllas que mejor desarrollan las destrezas y saberes implicados en las competencias.

- Un contexto de desempeño desafiante: en el que actualizar la competencia (una "zona de desarrollo próximo").

Como aspecto práctico a destacar para la formulación de competencias es, por un lado, la necesidad de no perder la dimensión de totalidad del proceso de enseñanza-aprendizaje, del sujeto y del contexto y, por el otro, la aplicación del sentido común y el saber profesional que harán un juicio equilibrado a la hora de tomar las decisiones pertinentes. Para facilitar este estado de alerta ante los riesgos de efectuar diseños incompletos, sesgados o inmediatistas se proponen las siguientes "alertas" conceptuales (García San Pedro, 2007): 
- Pragmatismo: enfatizar en exceso la dimensión del "saber hacer", sin que ese tipo de saber suponga y conlleve la reflexión, el saber estar, el saber ser. Por ejemplo: hacer ejercicios y prácticas reiterativas, sin progresión en la profundidad de las demandas ni vinculación en la trama curricular.

- Fragmentación: No considerar la naturaleza de la selección de competencias realizada. Desarticular su selección, formación y evaluación. La selección y trazado de las competencias debe tener un sentido, debe responder a un modelo de graduado deseado. Los componentes y fundamentos del proceso de enseñanza-aprendizaje deben responder a una dirección, a un diseño establecido colectivamente por los agentes educativos en forma participativa.

- Teoricismo: Enfatizar el saber qué, la dimensión potencial de la competencia, sin contemplar equilibradamente su dimensión actual, de efectiva realización. Este riesgo se observa en los perfiles altamente academicistas, diseños que dejan de lado el aspecto "más profesionalizador" de la profesión.

- Tecnicismo: Resaltar los aspectos técnicos, de habilidad en la ejecución, sin contemplar la relación con el juicio profesional, la dimensión de arte y creatividad en la ejecución. En este caso, se enfatiza la dimensión técnico-profesional dejando de lado el perfil o la posibilidad de desarrollar las competencias que promueven el desempeño académico y la continuidad en la formación reglada.

Estos aspectos señalados se proponen a título de reflexión, no pretenden abarcar ni establecer tipologías en los trazados curriculares. No obstante, como propuesta de mejora y de trabajo reflexivo del profesorado, sería interesante analizar y discutir el mapa de las competencias trazado para la titulación, a la luz de estas "alertas" conceptuales. Esta propuesta puede servir para establecer la consistencia del diseño curricular y realizar las modificaciones y ajustes necesarios según el criterio de los profesionales implicados en la formación.

\section{LAS COMPETENCIAS DESDE LA PERSPECTIVA DEL PROFESORADO. EL ESTUDIO DE CASOS}

\subsection{La metodología}

El estudio se organiza y realiza desde una perspectiva cualitativa. Se trata de conocer y comprender en profundidad lo que piensan los propios participantes en procesos formativos planificados por competencias y los significados que asignan al concepto de competencias.

Para Pérez Serrano (1994) y Merriam (1998), los estudios cualitativos son considerados un proceso activo, sistemático y riguroso de indagación dirigida, que permite tomar decisiones sobre lo investigable desde una orientación intersubjetiva y vinculada al objeto de estudio. Por su parte, Maykut y Morehouse (1999) sostienen que examinar las palabras y las acciones humanas con un método narrativo o descriptivo, representando así la situación como si los participantes la estuvieran experimentando. También se destacan las aportaciones de Sandín (2003) cuando identifica la metodología cualitativa como una actividad sistemática orientada a la comprensión en profundidad de fenómenos educativos y sociales, a la transformación de prácticas y escenarios socio-educativos, a la toma de decisiones y también hacia el descubrimiento y desarrollo de un cuerpo organizado de conocimientos. 


\subsection{Los casos y los informantes}

El diseño y desarrollo del estudio de casos múltiple desde la perspectiva hermenéutica siguió las orientaciones de Eisenhardt, (1989), Taylor y Bogdan (1987), Pérez Serrano (1994), Maykut y Morehouse (1999), Latorre, Rincón y Arnal, (2003), entre otros.

Los cuatro casos seleccionados bajo criterio de máxima variación pertenecen a titulaciones que participaron de la primera edición del "Pla Pilot de les Universitats Catalanes d'adaptació a l'Espai Europeu d'Educació Superior" (2004-2005) de la Agència per a la Qualitat del Sistema Universitari de Catalunya (AQU). Los informantes son responsables académicos y profesores implicados directamente en los cambios requeridos por el proceso de Bolonia.

\subsection{Los resultados}

Las preguntas que orientaron el estudio eran: ¿cómo ha sido percibido el cambio de modelo de enseñanza-aprendizaje? y ¿qué concepciones o ideas de "competencias" se asumen en las titulaciones? El tratamiento de la información obtenida se efectuó mediante análisis de contenido temático (Bardín,1986; Eisenhardt, 1989). El objeto no es identificar el estado de la implantación del grado en las titulaciones, sino describir qué concepciones y valoraciones se hallan entre los agentes del cambio. A continuación, se integran los resultados de la investigación.

\subsection{1. ¿Cómo ha sido percibido el cambio de modelo de enseñanza-aprendizaje?}

En relación a cómo ha sido percibido el cambio de modelo, el estudio encontró cuatro formas o visiones: como oportunidad de mejora de la calidad educativa, la confusión, la visión del cambio como fortalecimiento de la identidad de la titulación y las resistencias que genera.

- Primera visión: el cambio de modelo es oportunidad de mejora de la calidad educativa.

Esta visión se corresponde con una adecuación (sintonización) a las tendencias y estándares europeos y el desarrollo de mecanismos y sistemas que facilitan la transferencia y la comunicabilidad de los resultados. Es una forma de asegurar la calidad educativa al establecer modelos y criterios de seguimiento y la evaluación de las titulaciones que, hasta el momento, no se habían exigido ni aplicado en el contexto español. Algunos ejemplos de esta visión pueden observarse a través de los aportes de un profesor y un coordinador de titulación:

“...la universidad siempre se ha implicado mucho en este proceso, el rector anterior veía muy claro que esto era una oportunidad para mejorar la calidad de la docencia". CDHU1-En (4)

"Yo diría, que este cambio tendría que ser un cambio en profundidad, no un cambio de forma, sino que supone un replanteamiento de la formación de los profesionales que debemos formar, de la profesión, de los aspectos básicos... ” CDHU2-En (15)

- Segunda visión: el cambio de modelo genera confusión.

Esta visión engloba dos dimensiones, por un lado confusión entre el cambio en la vida institucional de la organización educativa en su propia evolución y la necesidad de responder a las nuevas estructuras derivadas de la convergencia europea, este aspecto genera confusión entre las personas entrevistadas al no diferenciarse claramente qué corresponde a cada dimensión. Por el otro lado, la confusión entre innovación docente vinculada al aspecto puntual de la innovación metodológica que se está extendiendo en las titulaciones y el cambio de modelo pedagógico universitario - prácticamente demanda mundial-, que supone el paso de la centralidad en la enseñanza a la centralidad 
en el aprendizaje a través de metodologías activas pero no sólo con ellas. Este enfoque hacia el "usuario" o destinatario de la formación universitaria no supone solamente una innovación -como proceso puntual y planificado- del aspecto metodológico, sino una nueva adecuación de todo el sistema.

"Porque yo creo que uno de los errores que se está cometiendo en la implementación de las pruebas piloto es en muchos casos la confusión de pruebas piloto con innovación docente." CDCT3-En (15)

"Innovación docente que quiere decir en este caso básicamente, aprendizaje cooperativo y uso de multimedia”. CDCT3-En (16)

“Lo del crédito europeo está bien porque aprendes otras metodologías.” PDHU11-En (34)

“Lo que pretendiamos era implantar o analizar o investigar con nuevas metodologías. ” CDHU2En (5)

En este caso, cabe resaltar dos consideraciones: por una parte, la vida institucional puede evolucionar, cambiar de estadios organizativos. Este cambio puede ser espontáneo o planificado, pero afecta a la organización. Por eso, se puede hablar de cambio de modelo de enseñanza-aprendizaje, porque cambia el foco y, en consecuencia, se afectan y modifican todas las estructuras. Cuando el cambio es planificado, el seguimiento de los procesos y la monitorización de las acciones posibilita una mayor integración de la nueva estructura al conjunto de la organización. En el problema de investigación que nos ocupa, no es posible aplicar un modelo de enseñanza centrado en el estudiante si no se parte de ver sus necesidades, si no se garantiza una diversidad metodológica que posibilite explorar las diferentes capacidades en pro del desarrollo de las competencias, por ejemplo, o si las agrupaciones de alumnos no favorecen contemplar la diversidad de los estudiantes. En consecuencia, se hace necesario adaptar la organización (titulación, en este caso) como un todo, es decir, hacer converger los esfuerzos, las estructuras físicas, los recursos humanos, el trabajo colaborativo y la cultura organizativa centrándolos en el estudiante.

En este sentido, es importante comprender la dimensión que tienen estos cambios para las personas implicadas, dado que conocer los valores, las inquietudes y posibilidades que se desvelan permite sentar las bases para la reflexión sobre el cambio, la dirección que puede tomar, sensibilizando a los implicados y alimentando las decisiones institucionales hacia la mejora. Por otra parte, las decisiones sobre las innovaciones permiten ver la importancia que tienen estas reflexiones cuando son construidas por la comunidad para el desarrollo del proceso de implantación. En definitiva, favorecer este tipo de reflexión es entender la posibilidad de transformar la realidad al concebir el cambio como proceso de definición, construcción y participación social.

- Tercera visión: cambio como fortalecimiento de la identidad.

Esta perspectiva remite a una revalorización de la cultura del centro, de los objetivos perseguidos por la titulación y del modelo educativo propuesto. Esta percepción se ha dado entre las titulaciones que venían realizando experiencias novedosas en distintos procesos o ámbitos y que, como respuesta a los cambios estructurales, han extendido la experiencia acumulada a toda la titulación, validando las decisiones tomadas y aprovechando esta coyuntura para evaluar el proceso. A nivel organizativo, se podría llegar a hablar de que han desarrollado estrategias de autorregulación, dado que, como resultado de sus evaluaciones, se ha fortalecido la dirección de las mejoras asumidas y se han reforzado aspectos ligados a la cultura y la identidad institucional. Estos rasgos se pueden observar en los casos en los que asumir el planteo de las competencias no supuso la preocupación inicial, sino que, por ejemplo, reforzaba las experiencias novedosas iniciadas previamente $\mathrm{o}$, dadas las necesidades de la titulación, se determinaba reforzar algunos aspectos como la formación del profesorado o la evaluación de los estudiantes. 
"No íbamos a cambiar ni la estructura de la titulación ni nada por el estilo. Hemos mantenido el número de créditos exactamente igual y lo único que hemos hecho es introducir, nuevos elementos, sobre todo en el ámbito de la evaluación." CDHU2-En (6)

"Aquí se planteó un proyecto educativo basado en competencias, que desde hace tres años se encuentra trabajando y elaborando las competencias finales que tienen que tener nuestros licenciados y esto es lo que va a marcar el rumbo de los futuros planes de estudio." CLCT7-En (1)

"Por lo tanto, nuestro proyecto no ha ido inicialmente detrás de las competencias, sino que se ha avanzado un poco..." CLCT8-En (3)

- Cuarta visión: las resistencias al cambio.

La falta de políticas educativas atrevidas y pioneras ha alimentado una percepción, bastante frecuente entre las personas entrevistadas, de miedo a un trabajo sin orientaciones claras, sin dirección o sentido, a un esfuerzo desmedido que, según sostienen los informantes, no tendrá recompensas ni reconocimiento académico-profesional. Esta falta de claridad conlleva la aparición de resistencias "la recomendación que se les dio fue que no se metieran a hacer un cambio tan grande" y desmotivación "no valdrá la pena tanto esfuerzo" que no favorecen el crecimiento ni el aprendizaje organizacional.

"Esto quiere decir que: depende de qué trabajemos ahora, el trabajo no va a poder ser aplicado posteriormente". CDHU2-En (16)

"En la prueba piloto todos improvisamos..., cuando digo todos no sólo son los profesores, sino los profesores, el centro, la universidad..., también la Generalitat y el Gobierno..., quiero decir, la prueba piloto presentada por el DURSI se ha improvisado bastante... Una improvisación que por una parte es criticable, pero que por la otra es comprensible." CDCT3-En (18)

"Se nos preguntaba por nuestra opinión sobre cómo debía realizarse el seguimiento de la prueba piloto... O sea, que fijate, que, cuando había pasado casi un cuatrimestre, se nos convoca por parte de los organizadores de la prueba piloto para preguntarnos cómo creíamos que debía hacerse este seguimiento." CDCT3-En (19)

"Yo tengo serias dudas de que se pueda transitar bien hacia el espacio europeo. Yo creo que una cosa es lo que debería ser la transición y otra cosa es lo que se está haciendo con ella". PDHU11-En (2)

"La intención de un grupo de profesores de cambiar radicalmente la metodología de trabajo y la recomendación que se les dio fue que no se metieran a hacer este cambio tan grande, pues los cursos les durarían dos años, después cambiará todo y no valdrá la pena tanto esfuerzo, pues no se sabe cómo cambiará." PDHU22-En (9)

En consecuencia, se puede decir que la primera visión hallada se relaciona con la dimensión externa del cambio -con la rendición de cuentas y la acreditación-, la segunda con el nivel conceptual -la necesidad de clarificar qué pasa, qué cambia y cómo se puede cambiar la organización-, la tercera con la identidad cultural de la titulación -quiénes somos, qué nos hace ser "buenos" o pioneros en esto, en qué nos consolidamos, qué "marca institucional" dejamos-y la cuarta con el currículum oculto del proceso de cambio -qué nos está frenando, qué temores tenemos, qué se dice y qué se debe hacer, quiénes tienen el poder y hacia dónde se debe ir-.

A modo de ejemplo, se ha dado el caso de titulaciones que han realizado innovaciones en sus áreas, previas a las demandas de cambio del Proceso de Bolonia y especialmente relacionadas con la centralidad del aprendizaje del estudiante (en metodologías de enseñanza y evaluación, en planes de acción tutorial, en el prácticum, pasantías, en formación del profesorado, etc.) y que, en consecuencia, han adaptado y cambiado sus estructuras. Por ejemplo, la introducción del Aprendizaje Basado en Problemas (ABP) en determinado curso; el "Plan de acción tutorial" enfatizando la orientación académica, la selección y elección de los trayectos formativos de los estudiantes; la integración de la figura del asesor pedagógico como facilitador y colaborador para el diseño de las asignaturas centrado en el estudiante. 
En otros casos, por el contrario, se ha hallado que las demandas de la convergencia europea han supuesto, a la par del cambio en las estructuras, una innovación en los procesos, la oferta, la modalidad de formación, la evaluación, etc. Es el caso de la realización de "cursos cero" realizados al inicio del curso académico como introducción y primeras orientaciones de cara a facilitar el desarrollo de competencias transversales. Otra modalidad son los cursos ofrecidos para el desarrollo de competencias genéricas - propuestos en paralelo al desarrollo del curso académico como refuerzo de determinadas competencias-.

Una tercera posibilidad se ha encontrado entre profesores que, por diversos motivos, han desarrollado de forma aislada experiencias novedosas en las asignaturas que no se han extendido a la titulación. Tras el seguimiento longitudinal de los casos, se ha podido comprobar que, actualmente, algunas de estas iniciativas, con la implantación de los nuevos grados, están caminando en paralelo, en otros casos han sido integradas y su experiencia aprovechada y extendida a los nuevos diseños.

Para concluir, es conveniente rescatar que la visión de las competencias, como modelo de formación para el ámbito universitario, rompe con la lógica más frecuente de las prácticas universitarias vigentes hasta ahora. En el estudio de casos, esto es percibido por los informantes como un valor en sí mismo debido a la "movilización" ocurrida en el profesorado, los programas, las estructuras.

"Hay algo en este proceso de innovación y es que ya no va a seguir como antes a nivel metodológico, de aula..." PLHU51-En (21)

“...quizás el valor de todo el proceso esté ahi, en que la gente se ha movido, de alguna manera." PLHU51-En (22)

\subsection{2. ¿Qué concepciones o ideas de "competencias" se asumen en las titulaciones?}

Como se ha mencionado anteriormente, el discurso teórico de las competencias ha generado varios posicionamientos en torno a sus supuestos, naturaleza, perspectivas de construcción, posibilidades y críticas. Por esta razón, la pregunta "What is competence?" formulada por Hager (1996), se presenta como cuestión central para comprender el punto de partida de este modelo en el ámbito universitario. ¿Qué concepto de competencias adoptan las titulaciones? ¿Es posible identificarlo? En consecuencia, se hace necesario conocer si las titulaciones lo han formulado y a qué definición han llegado. La respuesta a estos interrogantes sentará las bases para el trazado curricular.

\subsubsection{La conceptualización del constructo de "competencias"}

El concepto de competencias como "tema" aparece en las entrevistas de forma directa e indirecta, pero generalmente se da ligado a la demanda de clarificación conceptual. Esta clarificación tiene dos dimensiones: en relación al concepto mismo de competencias y al modelo de formación que se toma de referencia en la titulación.

Las referencias directas al concepto de competencias han surgido a raíz de que la falta de clarificación de un modelo de competencias acarrea la coexistencia de varios, con la consecuente dificultad de definir y unificar criterios. Concretamente, por ejemplo, se puede observar que en las listas de competencias coexisten diversos criterios de formulación, excesiva carga de la dimensión cognitiva (academicismo) o una desagregación de competencias que hace difícil la integración de las áreas para su desarrollo transversal.

"Lo que no podemos estar es lo que nos ha pasado, que por no discutir un modelo, cada vez se ha puesto uno diferente sobre la mesa, sin el suficiente trabajo sobre el propio modelo." PLHU51-En (7)

"Estamos trabajando sin definir el concepto y eso dificulta los niveles de comprensión y diálogo", "hay profesores que piden discutir el modelo, otros que valoran el proceso y ya se verá qué 
respuesta se da...", "por no definir el modelo, aqui hay cuatro modelos". "El clima es tenso, las opiniones muy divididas en cuanto a establecer los fundamentos, pero la pregunta es recurrente al no generarse el espacio necesario para formularla." (Extraído de las discusiones en reunión de profesores. Diario de Campo).

Esta falta de diálogo y acuerdo sobre un modelo a partir del cual establecer el perfil ha llevado a muchas confusiones, desgastes y discusiones que han obstaculizado el trabajo colegiado del profesorado. El siguiente es un registro a partir de la observación de un momento de fuerte tensión en una reunión de profesores:

"La falta de debate interno genera mucho malestar, el clima está encrispado, "así son las cosas y así salen de mal» dijo uno de los profesores” con tono exasperado. (Diario de Campo).

\subsubsection{Los fundamentos de las competencias}

Por otro lado, la pregunta por los fundamentos también se ha formulado de modo indirecto, estas referencias pueden ser clasificadas bajo tres formulaciones:

- Negación. Son frecuentes las siguientes expresiones: "una terminología (...) que proviene del campo de la Pedagogía", "puede dar la impresión de que en realidad no es más que eso, una terminología vacía que está de moda utilizar".

"Lo que pasa es que la discusión de... sí que hemos hecho cuando empezamos a definir competencias y a intentar explicarnos qué entendíamos, cada uno de nosotros sí que decía «yo hago, yo hago», pero sólo con el objetivo de definirla. La discusión sobre cómo la trabajo y para qué todavía no la hemos hecho, o sea, que, en ese sentido, no se ha producido cambio." CDHU2-En (48)

"Yo creo que ha habido falta de tiempo, falta de maduración para llegar a nuestro modelo, o por las prisas o por asumir las implicaciones que se ha tenidos que presentar." PLHU51-En (8)

"Pero sólo lo hemos hecho esta parte (definición de instrumentos para evaluación continua). La parte de competencias no, por eso te decía yo... no sabría decirte hasta qué punto te ayudaríamos porque esta parte de reflexión todavía no la hemos hecho y creo que es importante hacerla porque vamos a encontrar..." CDHU2-En (50)

"Creo que es importante no dejarlo al azar... Yo creo que ahora en muchos casos se está trabajando mucho las competencias transversales y es cierto y se ve la importancia." CDHU2-En (51)

"Si no se aclara, y viendo esta confusión, puede dar la impresión de que en realidad no es más que eso, una terminología vacía que está de moda utilizar, y eso es un gran peligro...” CDCT3En (3)

"Yo creo que la terminología esta se ha difundido ya, pero el contenido, se desconoce totalmente..." CDCT3-En (4)

- Reconocimiento sin concreción. Hay un reconocimiento de la necesidad de los fundamentos, pero que no se ha realizado por diversos motivos (tiempo o falta de acuerdo, por ejemplo) y se ha procedido a elaborar el "listado de competencias".

"Como estamos en fase de reformar los planes de estudio, no hemos definido las competencias, la idea será armar bloques de competencias. Pero ahora los profesores se encuentran asistiendo a reuniones de formación, pero no todos se implican de la misma manera." CDHU1-En (40)

- Reconocimiento de la dimensión operativa del trazado curricular. Reconocimiento efectivo de la necesidad de planteo conceptual, pero no en cuanto a las dimensiones del concepto o su naturaleza, sino en tanto acuerdo sobre qué se entiende operativamente por cada una y de qué modo se la trabaja. Lo interesante de esta perspectiva es el temor evidente de dar una definición acabada, definitiva, clara. Los silencios, las expresiones indirectas dan indicios de esta dificultad.

"Es un trabajo (...) no del todo resuelto, aunque ha habido madurez, aunque yo no estoy muy satisfecho con el producto final, el primer trabajo que habia que presentar, para hacer un pla- 
neamiento de formación basada en competencias era tener claro el perfil profesional, para quién se iba a hacer esa formación." PLHU51-En (2)

"Es un trabajo muy..., desde mi punto de vista, muy dificil de definir." CDHU2-En (17)

"Nos hemos dado cuenta de que teníamos un problema de terminología, de que cada uno de nosotros lee una cosa diferente detrás de cada competencia" CDHU2-En (18)

En cuanto al concepto de competencias en sí mismo, las definiciones son evasivas, generales, incompletas y confusas. Existen expresiones que son empleadas, con frecuencia, como sinónimos: habilidades-competencias, objetivos-competencias, actitudes-competencias. En el discurso, se pasa directamente de la competencia al perfil, no se evidencia el salto categorial.

"En el conjunto de preguntas que formulamos a los profesores hemos agregado lo que al final se han convertido en competencias transversales: ¿Cuáles eran las actitudes que creían que un alumno debia tener? ¿Qué eran necesarias para su asignatura?” PLCT72-En (10)

"No siempre es fácil diferenciar que hasta aquí llega la habilidad y hasta aqui la competencia, e incluso los textos teóricos que hay sobre esto a veces usan una palabra casi como sinónima. Si estamos hablando de competencia, por qué ahora me sale una palabra que es habilidad." PDHU21-En (14)

"Salieron, por ejemplo, capacidad de relación, integración, actitudes más genéricas, y tuvimos que diferenciar competencias generales de habilidades generales, por ejemplo, hacer una disolución, que es algo común a muchas asignaturas, no es una competencia transversal-porque eso implica más la actitud que toma el alumno-, sin embargo, también es transversal, porque implica a más de uno: entonces las distinguimos como transversales a unas materias y las generales eran fáciles porque las proponian todos lo profesores. ” PLCT72-En (11)

"Se confunden mucho las competencias con las capacidades y las capacidades con los objetivos, ese tipo de cosas." PLHU51-En (9)

"Si uno está dentro, también legitima lo que está haciendo de alguna manera y esa es la discusión que muchas veces mantenemos y, en algún caso, yo he dicho que algún profesor ha trasladado los objetivos de su asignatura al perfil profesional." PLHU51-En (5)

Con respecto a la dimensión normativa de la competencia (Stevenson, 1996), se la puede reconocer a través de dos formas: por un lado, en cuanto al referente valorado que suponen (al bien, lo bueno, en sentido aristotélico expresado por "lo vocacional") y, por el otro, en tanto referente de logros, de aspiración, de ordenación, que posibilita superar la "buena voluntad" individual para unificar criterios y diseñar estrategias comunes, es decir, que deja de lado el voluntarismo para legitimarse:

"Entonces, el hecho de plantear el logro de competencias finales implica un sentido vocacional, que ya está bien en si mismo, y un sentido de mayor objetividad: nosotros producimos alumnos $X$ y lo que se quiere es que sea el mejor. Lo que es seguro es que ya no pasará por la buena voluntad de algunos sino por un planteo más objetivo." PLCT72-En (27)

"Valor normativo, objetividad y transparencia, deja de lado al voluntarismo para legitimarse." (Diario de Campo).

También es interesante profundizar en el sentido que tiene el problema para los protagonistas. ¿Qué representaciones de la competencia se construyen? $\mathrm{Si}$, como propone Sandberg (2000), la concepción que los trabajadores tengan de su rol y de su trabajo ya es parte distintiva de la competencia y un camino de formación, es posible pensar que la percepción que tengan los formadores -en tanto trabajadores, enseñantes o promotores de las competencias- también incidirá en la formación que impartan, o sea, la formación por competencias. ¿Qué atribuciones de sentido se le da al concepto? ¿Qué significa para los educadores? En esta línea se evidencian tres significados de la competencia:

- Como referente de la excelencia "nosotros producimos alumnos $\mathrm{X}$ y lo que se quiere es que sea el mejor". 
"Uno no ha de demostrar a nivel teórico todo lo que se sabe, sino que hay que demostrar, saber actuar para que se sobresalga en los servicios que se puedan dar. Porque ya existian competencias y estudios sobre competencias y buenas, por lo menos en el campo de biblioteconomía." PDHU21-En (12)

"Yo creo que más elaborado hacia la excelencia en el servicio a la sociedad." PDHU21-En (13)

"Porque, de cara a las competencias, las necesidades también evolucionan. Una enciclopedia permanece siendo la misma, pero las necesidades de la gente cambian...” PDHU21-En (15)

- Como elemento integrador del saber y el hacer, la reflexión sobre la práctica, para unir el mundo universitario con el profesional.

"Competencia de que justamente puedas tener un discurso laboral que ilumine tu práctica y que puedas fundamentar el porqué haces esta opción y no otra. Ser un buen profesional no es sólo quien puede hacer bien una cosa, sino también quien sabe por qué lo hace de una manera y no de otra y por qué escoge esta metodología y no otra, por qué escoge este camino y no otro y qué hay detrás de todo estos, qué reflexión se puede hacer..., qué teoría, qué pensamiento... PLHU52- En (22)

"Realmente valoro que las competencias sirvan para pensar, para edificar curriculums que puedan favorecer la transferencia del conocimiento universitario a la práctica profesional..." PLHU52- En (27)

- Como una concepción más profunda del aprendizaje que va más allá de los objetivo de la asignatura.

"La competencia supone un nivel más profundo de aprendizaje, más global e integrado, es distinto a los objetivos de la asignatura. Quizás tú dices que tus objetivos especificos son muy claros, pero cuando se habla de competencias en un sentido más amplio, ¿cómo consigues en un trimestre que tus alumnos hablen con un vocabulario cientifico, por ejemplo, o que piensen según el método científico? Tú puedes colaborar y profundizar algunos aspectos, focalizar las actividades en ese sentido, pero no lo puedes lograr PLCT71-En (7)

\section{CONSECUENCIAS DEL CAMBIO DE MODELO}

En términos de diseño y desarrollo curricular, las competencias, como resultado de los aprendizajes, son el eje del cambio educativo. En consecuencia, la formación basada en competencias puede asumir el paso del énfasis en la enseñanza al aprendizaje, puesto que en su formulación va implicada la totalidad respondente de la persona como sujeto único, activo, en situación.

Este cambio en el enfoque curricular, reorienta las decisiones didácticas en relación con la enseñanza-aprendizaje y evaluación, centrándolas en el estudiante. Adam (2004) amplía las posibilidades de este enfoque al punto de señalar que facilita la integración con la formación profesional, la evaluación de aprendizajes previos, el desarrollo de cualificaciones para el aprendizaje permanente y el desarrollo de los sistemas de transferencia de créditos. En el contexto español, aún se está en los inicios del reconocimiento académico de los saberes y experiencias previas y se está avanzando en la transferencia entre los sistemas educativos, pero aún hay un largo camino por recorrer.

A corto plazo, se tratará de justificar el paso de la enseñanza y la evaluación centrada en el contenido y el docente al énfasis en el estudiante, sus procesos y resultados de aprendizaje. Como se ha presentado anteriormente, cada una de las visiones halladas representan dimensiones y matices del significado del cambio para la titulación. En conjunto, ofrecen, aunque no agotan, algunos matices que es interesante considerar a la hora de comprender el cambio que supone para las instituciones las demandas del Proceso de Bolonia. En particular, cada una aporta un aspecto propio que puede orientar el criterio para la aplicación 
de estrategias que favorezcan el desarrollo organizativo de la titulación y, paralelamente, el desarrollo profesional de los responsables de la formación.

En consecuencia, esta justificación debe ser entendida en sentido amplio, como cambio de modelo con un alcance profundo en sus ámbitos de diseño, gestión, metodologías y evaluación. Por tanto, como se ha presentado en los resultados, las distintas visiones del concepto de competencias enfatizan diversos aspectos que influyen en la trasposición didáctica de los trazados curriculares, $\mathrm{y}$, en consecuencia, en las prácticas de clase.

Si se parte de la concepción de la competencia como referente de la excelencia, los valores y aspiraciones institucionales (el "ethos") tendrán un peso muy alto a la hora de establecer cuáles son las competencias transversales. A su vez, representan un motivo significativo a la hora de unificar el trabajo del profesorado, pues el trazado curricular definitivo tendrá que "dar cuentas" de esos valores distintivos de la institución, ser identificados claramente y asumidos por todos los implicados (estudiantes, profesores y demás agentes educativos).

Por otro lado, si se parte de concebir la competencia como elemento integrador de la teoría y la práctica, el ámbito de incidencia de esta concepción se relaciona más directamente con el trazado curricular. Aquí adquiere un mayor protagonismo la dimensión pedagógico-didáctica de las asignaturas, puesto que las decisiones del profesorado en este sentido son las que marcan el rumbo de la integración -en ocasiones, dialéctica- teoríapractica, mundo académico-mundo profesional. En esta línea, es conveniente, por ejemplo, que las asignaturas del practicum o el trabajo final de carrera, sean concebidas a la luz de todo el trazado curricular, de modo que se concreten como la oportunidad de poner en práctica las competencias adquiridas desde la mirada del perfil profesional del futuro graduado. Por otro lado, el resto de las asignaturas o módulos no tienen que perder de vista que contribuyen a esta integración desde sus propuestas metodológicas diarias, por lo que se adelantan como escenarios previos para la puesta en escena final.

Finalmente, la visión de las competencias como una concepción más profunda del aprendizaje remarca la dimensión holística de este modelo de formación, haciendo ver que el contenido en sí mismo, sea de la naturaleza que fuera, no es el fin sino el medio, la oportunidad para proyectarlo dentro del saber-hacer profesional. Ya no es saber ser, saber hacer o saber qué, asiladamente, sino que es concretar esa integración en una conducta dirigida en un sentido más profundo: la sabiduría profesional, el ser ciudadano, el saber que el profesional deber ser y estar en el mundo y su compromiso deontológico trasciende el saber hacer aislado, descontextualizado. Esta visión se transmite a través de profesores comprometidos con su práctica docente, pues tiene componentes altamente vocacionales y motivacionales, y encuentra eco en una cultura institucional comprometida con la dimensión social de la profesión, abierta al contexto del que se nutre.

Las visiones halladas no son incompatibles, más bien se puede decir que ofrecen matices conceptuales complementarios, cada uno necesario en sí mismo por lo que representa, y útiles, pues movilizan aspectos de la vida institucional.

Con respecto a la gestión concreta e implementación de modelos de evaluación, por ejemplo, las auditorías de calidad realizadas en las universidades australianas (Barrie, 2006), se han centrado en las actuaciones que éstas realizan para que sus graduados adquieran efectivamente las competencias declaradas. En la mayoría de los casos, han revelado dos necesidades fundamentales. La primera, desarrollar currículos más y mejor orientados a las competencias genéricas y, la segunda, se refiere a la necesidad de contar con un mayor respaldo institucional para llevar adelante estas estrategias. En esta línea, existe otro riesgo: el hecho de realizar implementaciones estructurales con un alcance superficial -riesgo que se observó en los casos estudiados en García San Pedro (2007)-. 
Tras lo observado en el estudio de casos, uno de los desafíos relacionados con la calidad de la formación universitaria se encuentra tanto en la cuestión epistemológica como en reconocer la dimensión ontológica que suponen estos modelos en sí mismos. En medio de la vorágine que suponen los tiempos de acreditación de los nuevos grados, los nuevos diseños, las dificultades estructurales (Gairín et al., 2009b) observar las concepciones que orientan y definen el trazado curricular puede ayudar a gestionar mejor el esfuerzo y el trabajo del profesorado, lo que se traducirá también en mejorar la coherencia y la adecuación de las competencias con la formación ofrecida por las titulaciones.

En consecuencia, resulta interesante favorecer mecanismos y estrategias para que las universidades provean las evidencias necesarias de que sus procesos de enseñanza-aprendizaje responden a las competencias establecidas y de que sus estudiantes se hallan en condiciones de demostrar su adquisición (Bath et al., 2004). En el contexto español, se hace necesario seguir profundizando en los modelos de formación y evaluación por competencias que se desarrollan a raíz del Proceso de Bolonia y la concreción de los nuevos grados. Las reflexiones teóricas sobre el modelo educativo no se traducen a la práctica de forma significativa si no son acompañadas de un estudio profundo sobre las posibilidades de concretar la formación y evaluación por competencias en la universidad.

Analizar los modelos que se asumen, los matices que adquieren y los rasgos propios con los que la universidad española adopta el modelo de competencias es un desafío importante para comprender no sólo el cambio, sino también para valorar el esfuerzo del profesorado para llevarlo adelante. Así, por tanto, se insiste en que se favorezcan espacios de reflexión e investigación para subsanar el vacío epistemológico y metodológico desde el cual diseñar y monitorizar las mejoras que conlleva el modelo de formación por competencias.

\section{BILIOGRAFÍA}

ADAM, S., "Using Learning Outcomes" Report for the Bologna conference on learning outcomes. Edinburgh $1-2$ July, 2004.

BARDIN, L., El Análisis de contenido, Madrid: Akal, 1986.

BARRIE, S.C. (2005) Rethinking Generic Attributes. R. Landbeck (ed.) HERDSA News.

BARRIE, S.C. (2006). Understanding what we mean by the generic attributes of graduates. Higher Education, 2006, (51) 215-241.

BATH, D., SMITH, C., STEIN, S., \& SWANN, R., Beyond mapping and embedding graduate attributes: Bringing together quality assurance and action learning to create a validated and living curriculum. Higher Education Research and Development, 2004, 23 (3), 313-328.

BOWDEN J. et al., Generic Capabilities of ATN University Graduates Draft Report, January 2002.

CHANOCK, K., Challenges of the graduate attributes movement. En K. Deller-Evans \& P. Zeegers (Ed.), Proceedings of Fifth National Language and Academic Skills Conference. Adelaide: Flinders University, 2003.

CHANOCK, K., CLEREHAN R. y MOORE. T., Shaping university teaching towards measurement for accountability: Problems of the Graduate Skills Assessment test. Australian University Review, 2004, 47 (1) 22-29.

EISENHARDT, K.M., "Building Theories from Case-Study Research", Academy of Management Review, 1989, Vol: 14 (4) 532-550.

EUROPEAN COMISSION, ECTS Users' guide: European credit transfer and acumulation system and the diploma supplement. Directorate-General for Education and Culture, Bruselas, 2005.

GAIRÍN, J. "Estadios de desarrollo organizativo: de la organización como estructura a la organización que aprende", en Lorenzo, M. y otros (coord.) Enfoques comparados en Organización y Dirección de Instituciones Educativas. Granada: Grupo Editorial Universitario, 1999, Vol. 1: 47-91. 
GAIRÍN, J., ARMENGOL, C., GISBERT, M., GARCÍA SAN PEDRO, M.J., RODRÍGUEZ, D. y CELA, J.M. Guia per a l'avaluació de competències en l'àrea de Ciències Socials. AQU Catalunya, 2009a. Disponible en http://www.aqu.cat/doc/doc_14646947_1.pdf

GAIRÍN, J., GARCÍA SAN PEDRO, M.J., GISBERT, M., RODRIGUEZ GÓMEZ, D. y CELA, J.M. La evaluación por competencias en la universidad: Posibilidades y dificultades. Ministerio de Educación, Estudios y Análisis Ref. EA2008-0086, 2009b (en prensa).

GAIRÍN, J., La organización escolar. Contexto y texto de actuación. Madrid: La Muralla, 1996.

GARCÍA SAN PEDRO, M.J., Realidad y perspectivas de la formación por competencias en la universidad. Trabajo de Investigación. Departament de Pedagogia Aplicada, Universitat Autònoma de Barcelona. 2007. Disponible en: http:/hdl.handle.net/2072/8999 .

HAGER, P., HOLLAND, S. \& BECKETT, D., "Enhancing the learning and employability of graduates: the role of generic skills", BHERT Position Paper. B-HERT, Melbourne, July, 2002, $\mathrm{N}^{\circ} 9$.

HUSSEY, T. \& SMITH, P., "Learning outcomes: a conceptual análisis", Teaching in higher education, 2008, 13, (1)107-115.

KENNEDY, D., HYLAND, A. \& RYAN, N., "Writing and using learning outcomes: a practical guide", Froment,E., Kohler, J. \& Purser, L. and L. Wilson (eds.): EUA Bologna Handbook-Making Bologna Work, Raabe Verlag Berlin, 2006, C 3.4-1.

KIFT, S. "Harnessing Assessment and Feedback to Assure Quality Outcomes for Graduate Capability Development: A Legal Education Case Study", AARE Conference, Brisbane, December, 2002.

LEVI-LEBOYER, C., La Gestión de las Competencias, Ediciones Gestión 2000, Barcelona, 1997

MAYKUT, P. y MOREOUSE, R., Investigación cualitativa. Barcelona: Hurtado, 1999.

MCMILLAN, J.H. y SCHUMACHER, S. Investigación Educativa. (5 edic), Madrid: Pearson Educación, 2007.

MILES, B., y HUBERMAN, A., Qualitative data analysis. A sourcebook of new methods. Beverly Hills: Sage, 1984.

PÉREZ SERRANO, G., Investigación cualitativa. Retos e interrogantes I y II. Madrid: La Muralla, 1984.

SANDÍN, M. La investigación cualitativa en educación, Madrid: McGraw-Hill, 2003. 